
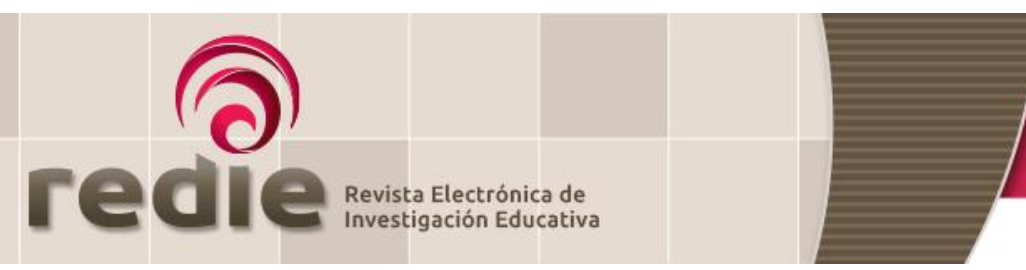

Vol. 20, Núm. 1, 2018

\title{
El proceso de enseñanza y aprendizaje de las Matemáticas. Concepciones de los futuros profesores del sur de Chile ${ }^{1}$
}

\section{The Teaching and Learning Process in Mathematics. Perceptions of Future Teachers in Southern Chile}

\author{
Miguel Friz Carrillo (1) mfriz@ubiobio.cl \\ Rodrigo Panes Chavarría (2) rpanes@ubiobio.cl \\ Pedro Salcedo Lagos (3) psalcedo@udec.cl \\ Susan Sanhueza Hernández (2) ssanhueza@ucm.cl \\ (1) Universidad del Bío-Bío \\ (2) Universidad Católica del Maule \\ (3) Universidad de Concepción \\ (Recibido: 20 de junio de 2016; Aceptado para su publicación: 28 de noviembre de 2016)
}

Cómo citar: Friz, M., Panes, R., Salcedo, P. y Sanhueza, S. (2018). El proceso de enseñanza y aprendizaje de las Matemáticas. Concepciones de los futuros profesores del sur de Chile. Revista Electrónica de Investigación Educativa, 20(1), 59-68. https://doi.org/10.24320/redie.2018.20.1.1455

\section{Resumen}

El propósito del estudio fue analizar las concepciones que poseen estudiantes de Pedagogía del primer y último año de titulación ( $n=50)$ hacia la enseñanza de las Matemáticas a partir de tres dimensiones: 1) las matemáticas como objeto de estudio, 2) utilidad de las matemáticas y 3) enseñanza y aprendizaje de las matemáticas. Utilizando un enfoque cuantitativo de diseño descriptivo y comparativo, los resultados demuestran el predominio de una concepción de las matemáticas como una disciplina instrumental asistida principalmente por el uso de textos escolares en los estudiantes de primer año, aspecto que es modificado en el último curso, cuando se integran elementos culturales y comunicativos de las matemáticas, así como un carácter interdisciplinario. En la dimensión enseñanza y aprendizaje de las matemáticas no se observaron diferencias entre grupos, elemento que plantea una serie de desafíos para la formación inicial de profesores.

Palabras clave: Formación de profesores, enseñanza de las matemáticas, enseñanza, aprendizaje, concepciones.

\section{Abstract}

The purpose of the study was to analyze the perceptions of first and last-year pedagogy students ( $\mathrm{n}=50$ ) regarding mathematics teaching from three dimensions: 1 ) mathematics as an object of study, 2) the utility of mathematics, and 3) teaching and learning mathematics. A quantitative approach was used with

\footnotetext{
1 Proyecto Fondecyt 1140457 "Plataforma adaptativa online para el fortalecimiento de las competencias matemáticas y pedagógicas a partir del estudio léxico semántico de estudiantes y profesores de pedagogía en matemática" de la Comisión Nacional de Investigación Científica y Tecnológica de Chile (Conicyt). Con la colaboración del Grupo de Investigación en Educación y Educación Matemática Gl 152823/EF y Proyecto DIUBB 153523 3/I.
} 
a descriptive-comparative design, and results show the prevalence of an understanding of mathematics as an instrumental discipline mainly supported by the use of textbooks in first-year students. This changes by the last year of studies, when cultural and communicative aspects are incorporated into mathematics, which takes on an interdisciplinary nature. No differences between groups were observed in the learning and teaching dimension of mathematics, which raises a series of challenges for teachers' initial training.

Keywords: Teacher education, mathematics education, teaching, learning, perceptions.

\section{Introducción}

El trabajo en aula del profesor, caracterizado didácticamente por la tríada estudiante-profesor-saber, ha constituido uno de los pilares sobre el cual se explican las interacciones al interior de la sala; sin embargo, las investigaciones de Díaz (2006), Mato, Espiñeira y Chao (2014) y Panes y Parra (2013) dan cuenta de la existencia de prácticas que sólo pueden ser comprendidas en función de las orientaciones y concepciones que las orientan, así como del entorno en el que se desarrollan.

La dimensión afectiva de las matemáticas (Gamboa, 2014; Gómez-Chacón, 2000) plantea que no sólo el componente del conocimiento de la disciplina juega un papel fundamental en el éxito de los estudiantes en la asignatura, sino que existe una serie de factores (concepciones, emociones, creencias, actitudes y valores en el desarrollo) que pueden explicar dicha relación. Desde el impulso logrado en los años ochenta el constructo ha sido desarrollado y estudiado, es así como líneas de investigación al interior de la educación matemática -como la dimensión afectiva de las matemáticas (Gómez-Chacón, 2010) o los aportes educativos del Teacher thinking (pensamiento del profesor) (Baena, 2000) y desde el enfoque de las teorías implícitas o subjetivas (Makuc y Larrañaga, 2015)- las han considerado en sus investigaciones.

Para Thompson (1992), las concepciones son una estructura mental general que abarca creencias, significados, conceptos, imágenes mentales, preferencias y gustos; mientras Pehkonen (2006) las define como "creencias conscientes". Por su parte, García, Azcárate y Moreno (2006) señalan que en el caso del profesor las concepciones consisten en la estructura que cada docente da a sus conocimientos para luego enseñarlos o trasmitirlos a sus estudiantes. En su relación con la naturaleza de la disciplina, Mora y Barrantes (2008) consideran que las concepciones se pueden identificar con la percepción o visión que se tiene con respecto a ellas.

Considerando su actuar y caracterización, Friz, Sanhueza, Sánchez, Sámuel y Carrera (2009) plantean que en el caso de docentes y estudiantes las concepciones sobre el valor de los contenidos y los procesos propios del aula llevan a la interpretación, a la decisión y al actuar; así, estos elementos se verificarían en la toma de decisiones de textos, de estrategias de enseñanza y de la evaluación. De corpus, García, Azcárate y Moreno (2006) presentan algunos elementos que caracterizan estas concepciones: 1) forman parte del conocimiento, 2) son producto de las formas de entender, 3) actúan como filtros ante decisiones y 4) influyen en los razonamientos.

Sobre cómo se forman y desarrollan las concepciones, Gil, Blanco y Guerrero (2005) y Gómez-Chacón (2010) coinciden en que tienen su origen en la experiencia, en la observación directa y en las informaciones que son recibidas y asimiladas. Debido a ese carácter dinámico que involucra decisiones implícitas y explícitas, Friz, Sanhueza y Figueroa (2011) consideran que las concepciones se configuran en un conjunto estructurado de ideas, axiologías, ontologías, epistemologías y metodologías. Por su parte, Catalán (2011) plantea que las concepciones forman parte del conocimiento profesional del docente, construidas sobre la base de conocimientos pedagógicos históricamente elaborados y trasmitidos durante la formación y la práctica pedagógica (Cárcamo y Castro, 2015).

En su investigación, Barrón (2015) encuentra que las concepciones epistemológicas presentes en profesores, referidas a concepciones globales y personales en torno a lo educativo, lo didáctico, la disciplina y sus prácticas tienen diversas derivaciones que se plasman en el currículo. En ese sentido, en términos de actitudes que se promueven, coincide con Sanhueza, Friz y Quintriqueo (2014) al considerar las expectativas e informaciones que se reciben de parte del profesor como un elemento que los alumnos perciben de sus profesores. En su estudio, Briceño y Benarroch (2013) dan cuenta que al interior de 
formadores en ciencias abundan creencias empiristas en contraposición a concepciones constructivas de la propia ciencia, así como perspectivas reduccionistas y un desconocimiento de los procesos de enseñanza, advierten sobre la necesidad de abordarse en un proceso de formación de profesores, debido a que la cultura escolar y la escolarización, según Prieto y Contreras (2008), facilitan la construcción social de creencias con respecto a la educación, la enseñanza, el aprendizaje, la evaluación y la disciplina.

Al estudiar las concepciones con respecto al aprendizaje de un grupo de estudiantes de primero y quinto grado universitario, utilizando situaciones presentadas a modo de dilemas y haciendo la comparación con docentes sobre 15 años de experiencia, Cárcamo y Castro (2015) dan cuenta de que no existen muchas diferencias entre las concepciones de los estudiantes; es decir, no presentan diferencias en torno a alguna concepción de aprendizaje, aunque se muestre una mayor inclinación hacia perspectivas constructivistas.

Los trabajos expuestos hasta ahora proporcionan elementos de discusión sobre las concepciones que se tienen de las matemáticas; sin embargo, las diferencias entre quienes se están iniciando como profesores y aquellos que están prontos a serlo no han sido suficientemente documentadas. Planteamos como supuesto que los estudiantes de primer año de formación traen consigo una serie de concepciones más vinculadas a sus experiencias como aprendices, mientras que se esperaría que aquellos que han recibido formación en competencias pedagógicas y didáctica de las matemáticas durante su carrera hayan modificado sus concepciones iniciales.

A partir de los antecedentes expuestos, el objetivo de este trabajo es analizar las concepciones que manifiestan futuros profesores de matemáticas acerca de esta disciplina, el proceso de enseñanza y aprendizaje, y su utilidad para la vida cotidiana, diferenciando las respuestas según el año de formación en el que se encuentran.

\section{Metodología}

El trabajo tiene un enfoque metodológico cuantitativo de diseño no experimental tipo encuesta (Gay y Airasian, 2000); la información se obtuvo a través de una escala tipo Likert creada y administrada a futuros profesores en formación, estudiantes de una universidad pública del sur de Chile.

La formación de profesores tiene una duración de 4 a 5 años y su ingreso es mediante una prueba de selección universitaria. En el estudio participaron 50 estudiantes de una universidad pública regional del sur de Chile: $50 \%$ de primer año y $50 \%$ de quinto año (64\% de la muestra eran mujeres), cuyas edades fluctuaban entre 18 y 29 años.

Se diseñó un cuestionario ad hoc para tener una aproximación a las concepciones hacia las matemáticas. La parte I contenía variables demográficas: curso, género y edad; la parte II incluyó 25 ítems referidos a la enseñanza y aprendizaje de las matemáticas a los que se debía responder expresando el grado de acuerdo (1=Muy en desacuerdo a 4=Muy de acuerdo). Los análisis psicométricos del instrumento arrojaron un índice de validez de contenido (IVC) de 0.85, mientras que la fiabilidad por consistencia interna arrojó un alpha de Cronbach de 0.738, datos considerados adecuados para el propósito del estudio (Cornejo, Sanhueza y Rioseco, 2012). Las dimensiones (variables) obtenidas luego del análisis factorial fueron:

- Concepciones hacia la Matemática (сM). Hace referencia a valores afectivos que se atribuyen a la disciplina; consta de 7 variables, un ejemplo es la R22: "La matemática se rige sólo de reglas y operaciones".

- Concepciones acerca de la Utilidad de las Matemáticas (CUM). Hace referencia al grado de valoración sobre las actividades en las que influyen las matemáticas; consta de 7 variables, ejemplo de esta dimensión es la R24: "Las matemáticas son importantes para todas las asignaturas de los alumnos". 
- Concepción sobre el proceso de Enseñanza y Aprendizaje de las Matemáticas (CAM). Integra reactivos que valoran situaciones sobre cómo se aprende y enseñan las matemáticas; consta de 12 variables, como la R26: "Las matemáticas se aprenden por predisposición natural del alumno" y la R18: "Las matemáticas se deben enseñar guiadas por el libro de texto".

El cuestionario fue aplicado por dos encuestadores durante las sesiones regulares de los cursos, en ambas aplicaciones se informó a los participantes de los objetivos del estudio y los responsables del mismo, y se les solicitó firmar un consentimiento informado. Durante los 20 minutos en promedio que duró la aplicación se dio asistencia y se proporcionaron los materiales necesarios.

El análisis de datos consideró técnicas descriptivas e inferenciales. Haciendo uso de PASW18 se calcularon medidas de tendencia central (Media, Moda), medidas de dispersión (Desviación típica), y se obtuvieron frecuencias y porcentajes para cada variable del estudio. Para la búsqueda de diferencias estadísticas significativas entre los estudiantes de distinto curso se utilizó la prueba $t$ para comparación de medias entre grupo trabajándose con una $\alpha=0.05$.

\section{Resultados}

Los resultados obtenidos en la aplicación del instrumento se presentan en dos momentos diferenciados, en una primera instancia se muestran aquellos obtenidos para el total de la muestra, en una segunda instancia se presenta un análisis de comparación de medias en función del curso del encuestado y los resultados por dimensión.

Los valores medios y la desviación típica de las variables correspondientes a las dimensiones CM, CUM y CAM para el total de la muestra ( $\mathrm{N}=50)$, así como los porcentajes de respuestas, se muestran en la tabla I. Considerando estos datos se realiza el posterior análisis descriptivo. 
Tabla I. Concepciones hacia las Matemáticas, su Utilidad y el proceso de Enseñanza y Aprendizaje

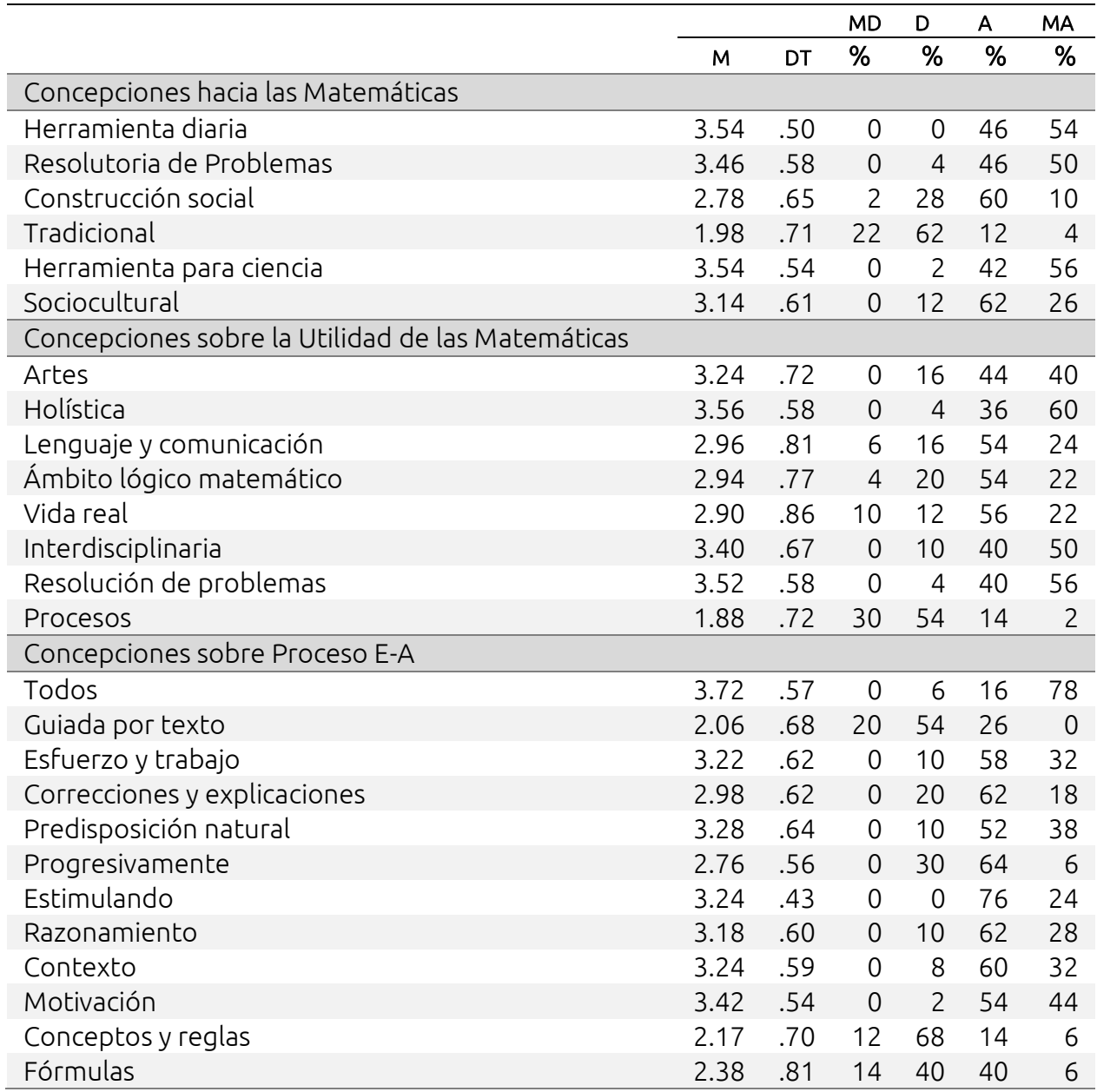

M=Media, DT=Desviación Típica, MD=Muy en Desacuerdo, D=En Desacuerdo, A=De Acuerdo, MA=Muy de Acuerdo.

Fuente: Elaboración propia.

En términos generales las concepciones más valoradas por los estudiantes en la dimensión Concepción hacia las Matemáticas (CM) fueron aquellas que consideran a las matemáticas como una ciencia que ayuda en el transcurso de la vida a la solución de problemas cotidianos ( $M=3.54 ; \mathrm{DT}=0.50)$, donde $54 \%$ de los encuestados está "muy de acuerdo" y $46 \%$ está "de acuerdo". Y aquella en la que los estudiantes estiman que las matemáticas proveen de herramientas necesarias para el trabajo en otras ciencias ( $M=3.54$; DT=0.54), con similares porcentajes, $56 \%$ de los encuestados estuvo "muy de acuerdo" y $42 \%$ marcó la opción "de acuerdo". La visión tradicional de las matemáticas regidas por reglas y operaciones (M=1.98; $\mathrm{DT}=0.71$ ) fue valorada negativamente por el $84 \%$ de los estudiantes. Los valores formativos, cultural e histórico que promueve $(M=3.14 ; D T=0.61)$ y el papel asignado como una ciencia capaz de resolver problemas de la vida real ( $M=3.46 ; \mathrm{DT}=0.58)$ obtuvieron altas puntuaciones.

En la Dimensión Utilidad de las Matemáticas (CUM) se manifiesta una alta aceptación de los encuestados al considerar que las matemáticas son útiles y necesarias en todos los ámbitos de la vida (M=3.56; DT=0.58), sólo $4 \%$ de la muestra estuvo "en desacuerdo". Su utilidad e interdisciplinariedad con todas las asignaturas es altamente valorada $(M=3.40 ; D T=0.67)$, así como la importancia de enseñarla como medio para la resolución de problemas ( $M=3.52 ; \mathrm{DT}=0.58)$. En su relación con el arte y la música $(M=3.24 ; \mathrm{DT}=0.72)$ y la asignatura de Lenguaje y comunicación (M=2.96; DT=0.81) el estudiante encuentra nexos vinculantes, aunque en menor medida. 
La dimensión sobre el proceso de Enseñanza y Aprendizaje de las Matemáticas (CAM) fue evaluada con 12 variables, destaca por su amplia valoración el que todos podemos aprender matemáticas ( $M=3.72$; DT=0.57), 78\% está "muy de acuerdo" con esa concepción. En relación con el aprendizaje de las matemáticas las concepciones más representativas son aquellas que apelan al aprendizaje mediante el esfuerzo y trabajo personal ( $M=3.22$; $D T=0.62$ ), las que estimulan y fomentan procesos cognitivos ( $M=3.24$; $\mathrm{DT}=0.43$ ) y el esfuerzo y trabajo personal $(M=3.22$; $\mathrm{DT}=0.62)$, actividades propias del aprendizaje. Teniendo en cuenta la actividad de enseñar, un alto porcentaje (98\%) considera que las actividades más adecuadas para enseñar son las que destacan motivación e interés por parte de los alumnos ( $M=3.42$; $D T=0.54)$; la vinculación contextualizada de situaciones reales en la enseñanza ( $M=3.24$; DT=0.59) junto a actividades que apelan al razonamiento y al análisis obtuvieron también alta valoración.

Los menores valores obtenidos corresponden a actividades propias de un modelo tradicional de enseñanza y aprendizaje de las matemáticas, ligado fuertemente a la institución escolar, como el aprendizaje memorístico de conceptos, fórmulas y reglas ( $M=2.17$; $\mathrm{DT}=0.70)$; la aplicación de fórmulas previamente explicadas por el profesor $(M=2.38$; $D T=0.81)$ o la consideración de capacidades especiales para aprender matemáticas ( $M=2.76$; DT=0.56) obtienen baja puntuación.

\subsection{Diferencias en las concepciones a partir del nivel/curso formativo}

Con el propósito de buscar diferencias estadísticamente significativas entre los grupos se utilizó la prueba $t$ de Student, en consideración de los supuestos previos y los valores obtenidos se analiza por cada dimensión la comparación resultante en función del curso del estudiante.

Tabla II. Comparación de medias en función del curso, Dimensión cM

\begin{tabular}{lcccc}
\hline $\begin{array}{l}\text { Concepciones } \\
\text { hacia las Matemáticas }\end{array}$ & \multicolumn{4}{l}{ Prueba $t$} \\
\hline & \multicolumn{1}{c}{$t$} & $\mathrm{Gl}$ & sig & Dirección \\
\cline { 2 - 5 } Herramienta diaria & -2.028 & 48 & .048 & $\mathrm{P}<\mathrm{Q}$ \\
Resolutoria de problemas & -1.228 & 48 & .226 & \\
Construcción social & -3.071 & 46 & .004 & $\mathrm{P}<\mathrm{Q}$ \\
Tradicional & 2.269 & 48 & .028 & $\mathrm{P}>\mathrm{Q}$ \\
Herramienta para ciencia & -3.111 & 48 & .003 & $\mathrm{P}<\mathrm{Q}$ \\
Sociocultural & -2.177 & 48 & .034 & $\mathrm{P}<\mathrm{Q}$ \\
\hline
\end{tabular}

Dirección P=Alumnos de Primer Año, Q=Alumnos de Quinto Año. Fuente: Elaboración propia.

Los resultados muestran que durante el primer año de formación los estudiantes reconocen en las matemáticas una rigidez de reglas y operaciones $[t(48)=2.27 ; p=0.028]$ al constituirse como ciencia, esta situación podría explicarse por la formación escolarizada que han enfrentado en años recientes. A su vez, se evidencia que los reactivos asociados a perspectivas de corte sociocultural y constructivista de las matemáticas, por ejemplo, que las matemáticas son construidas socialmente [t(46)=-3.07; $p=0.004]$, las matemáticas promueven un valor formativo, cultural e histórico [t(48)=-2.18; $p=0.034]$, las matemáticas, como ciencia, nos ayudan en el transcurso de nuestra vida a la solución de problemas cotidianos $[t(48)=2.03 ; p=0.048]$, muestran diferencia a favor de los estudiantes de quinto año que han obtenido su licenciatura en educación que los habilita como futuros profesores. Se encontraron, además, diferencias estadísticamente significativas en el reactivo "Las matemáticas nos proveen de herramientas para el trabajo en otras ciencias" [t(48)=-3.11; $p=0.003]$ a favor de los estudiantes de quinto grado. 
Tabla III. Comparación de medias en función del curso, Dimensión cum

\begin{tabular}{|c|c|c|c|c|}
\hline \multirow[t]{2}{*}{$\begin{array}{l}\text { Concepciones } \\
\text { sobre la Utilidad de la Matemática }\end{array}$} & \multicolumn{4}{|c|}{$\begin{array}{l}\text { Prueba } t \\
\text { para igualdad de medias }\end{array}$} \\
\hline & $t$ & $\mathrm{Gl}$ & sig & Dirección \\
\hline Artes & -2.493 & 48 & .016 & $\mathrm{P}<\mathrm{Q}$ \\
\hline Holística & -2.589 & 42 & .013 & $P<Q$ \\
\hline Lenguaje y comunicación & -4.007 & 48 & .000 & $P<Q$ \\
\hline Ámbito lógico matemático & .162 & 48 & .872 & \\
\hline Vida real & -.549 & 48 & .585 & \\
\hline Interdisciplinaria & -2.191 & 48 & .033 & $\mathrm{P}<\mathrm{Q}$ \\
\hline Resolución de problemas & -2.010 & 48 & .050 & $\mathrm{P}<\mathrm{Q}$ \\
\hline
\end{tabular}

Dirección P=Alumnos de Primer Año, Q=Alumnos de Quinto Año.

Fuente: Elaboración propia.

A partir de la lectura de los resultados de esta segunda dimensión se infiere que a mayor nivel de formación para profesor el estudiantado relaciona y valora significativamente la utilidad de las matemáticas en diversas áreas; por ejemplo, al relacionarlas con las creaciones artísticas como el arte y la música [t(4)=-2.49; $p=0.016]$ o vinculándola a asignaturas como el Lenguaje y comunicación [t(48)=-4.01; $p=0.000]$, situación que se refuerza cuando se considera que las matemáticas son útiles y necesarias en todos los ámbitos de la vida [t(42)=-2.589; $p=0.013$ ] e importantes para todas las asignaturas de los alumnos [t(48)=-2.191; $p=0.33]$. Otra diferencia significativa a favor los estudiantes con más formación docente hace referencia a la enseñanza y promoción de las matemáticas, ya que "ayudan a resolver problemas de la vida" $[t(48)=-2.01 ; p=0.05]$.

Tabla IV. Comparación de medias en función del curso, Dimensión CAM

\begin{tabular}{lcccc}
\hline \multirow{2}{*}{ Concepciones sobre Proceso E-A } & \multicolumn{4}{l}{ Prueba } \\
& para igualdad de medias \\
\hline & \multicolumn{1}{c}{$t$} & Gl & sig & Dirección \\
\cline { 2 - 5 } Todos & -.490 & 48 & .626 & \\
Guiada por texto & -.618 & 48 & .540 & \\
Esfuerzo y trabajo & .227 & 48 & .821 & \\
Correcciones y explicaciones & .678 & 48 & .501 & \\
Predisposición natural & .882 & 48 & .382 & \\
Progresivamente & .505 & 48 & .616 & \\
Estimulando & -.652 & 48 & .518 & \\
Razonamiento & -1.694 & 48 & .097 & \\
Contexto & -5.407 & 47 & .000 & P<Q \\
Motivación & -.260 & 48 & .796 & \\
Conceptos y reglas & 1.010 & 48 & .317 & \\
Fórmulas & .523 & 48 & .603 & \\
\hline
\end{tabular}

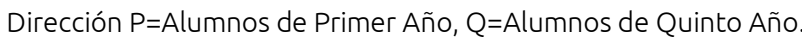

Fuente: Elaboración propia.

Tras analizar los resultados en función de las medias en esta dimensión, los resultados muestran diferencias estadísticas significativas a favor de los estudiantes de quinto año, que están "muy de acuerdo" con que las actividades más adecuadas para enseñar matemáticas son las que destacan la utilidad y conexión con situaciones reales $[t(47)=-5.407 ; p=0.00]$. En las restantes variables de la dimensión no es posible encontrar diferencias estadísticamente significativas a favor de uno u otro curso.

\section{Discusión y conclusiones}

En términos metodológicos, la escala CM se presenta como un instrumento adecuado para los propósitos del estudio, así lo demuestran las propiedades de validez y fiabilidad reportados en el trabajo. Teniendo en cuenta los resultados generales obtenidos en la aplicación se evidencia que al ingresar a una escuela de formación de profesores, los estudiantes traen una carga de apreciaciones, ideas y creencias sobre las matemáticas, su utilidad y la forma de abordar los procesos de enseñanza y aprendizaje, producto de sus 
experiencias como escolares y su vinculación y trabajo con las matemáticas, resultados coincidentes con los obtenidos por Friz et al. (2011) y Mato et al. (2014).

En términos generales los estudiantes tienen una valoración de las matemáticas como disciplina instrumentalista (Ernest, 1998) y reconocen su carácter interdisciplinario. Asimismo, reconocen el carácter socio-cultural de las matemáticas, aspecto que también es incluido en los programas oficiales atribuyendo un valor formativo, cultural e histórico (Ministerio de Educación [Mineduc], 2009).

Con relación a las concepciones más tradicionales de las matemáticas, ligadas principalmente a modelos epistemológicos euclideanos y cuasi-empíricos (Gascón, 2001) se recogen bajos valores medios, es decir, concepciones ligadas a la enseñanza y aprendizaje que apelan al uso del texto como medio para guiar la enseñanza, aquellas que consideran como única verdad el seguimiento de pautas y reglas previamente pasadas y explicadas por el profesor son infravaloradas.

Respecto de los análisis comparativos por nivel formativo, se concluye que son los estudiantes de último año de carrera (quinto) los que otorgan un efecto pragmático a la enseñanza de las matemáticas, relacionando su uso y aplicabilidad a diferentes situaciones cotidianas, lo que podría explicarse por el tiempo empleado en el estudio de las matemáticas, por ejemplo, Aritmética, Cálculo, Álgebra, Geometría, Estadística, y también asignaturas del área pedagógica, como Currículo, Evaluación, Gestión, Didáctica, además de la línea de prácticas. Esta formación les ha permitido atribuir mayores utilidades al campo de conocimiento de las matemáticas, y ha configurado una mayor amplitud del rango de aplicabilidad de la disciplina. Estos resultados son coincidentes con el trabajo de Sanhueza, Penalva y Friz (2013), quienes destacan que las competencias que adquieren una mayor relevancia para los futuros maestros luego de un experimento de enseñanza son la dimensión social de las matemáticas y los procesos de interacción y comunicación. Por otra parte, también es posible explicar estos resultados a la luz del trabajo realizado por Horn (2008), quien argumenta que los aprendizajes sobre la enseñanza de las matemáticas que van incorporando futuros profesores no se deben al resultado de acciones educativas de profesores individuales, sino que emergen de la interacción entre las identidades en desarrollo de los estudiantes y los mundos matemáticos que existen en las instituciones, en este caso, la universidad.

Por el contrario, los resultados muestran que en los estudiantes de primer año predomina una concepción más tradicional de las matemáticas (de reglas y operaciones), aspecto que podría explicarse por las metodologías de enseñanza que en palabras de Aravena, Caamaño y Giménez (2008) adolece de estrategias diversificadas que favorezcan formas de pensar matemáticamente, como lo es la resolución de problemas. Para Sánchez et al. (2006) los resultados se explican porque los futuros profesores combinan conocimiento desde dominios diferentes (por ejemplo, sus propias experiencias) para interpretar el contenido matemático y aprendizaje matemático, esto los llevaría a reproducir modelos dominantes de corte tradicional.

Esta idea es compartida por Aravena y Caamaño (2007), quienes manifiestan que los dispositivos didácticos bajo los cuales operan inicialmente los estudiantes responden a los modelos escolares que fueron formados los futuros profesores, que resultan difíciles de corregir, además de la falta de articulación entre temas matemáticos y otras áreas del conocimiento.

Con el instrumento no fue posible constatar diferencias estadísticamente significativas entre los participantes en la CAM, siendo un aspecto interesante de abordar en investigaciones futuras, por ejemplo, a través de entrevistas u observaciones de clases.

Por último, creemos que la formación de profesores con énfasis en lo disciplinar, didáctico y pedagógico puede influir en las ideas, concepciones y creencias que el futuro profesor va adquiriendo consciente e inconscientemente, y que posteriormente reproducirá en el aula. Sánchez, Díaz, Sanhueza y Friz (2008) señalan que los futuros maestros transferirán a los centros escolares aquello que hayan experimentado y vivido en su formación inicial universitaria, lo que se traduce en términos de políticas como un desafío y una propuesta para ser abordada en la formación inicial del profesor. 


\section{Referencias}

Aravena, M. y Caamaño, C (2007). Modelización matemática con estudiantes de secundaria de la comuna de Talca, Chile. Estudios Pedagógicos, 33(2), 7-25.

Aravena, M., Caamaño, C. y Giménez, J. (2008). Modelos matemáticos a través de proyectos. Revista Latinoamericana de Investigación en Matemática Educativa, 11(1), 49-92.

Baena, M. (2000). Pensamiento y acción en la enseñanza de las ciencias. Enseñanza de las Ciencias, 18(2), 217-226.

Barrón, C. (2015). Concepciones epistemológicas y práctica docente. Revista de Docencia Universitaria, 3(1), 35-56.

Briceño, J. y Benarroch, A. (2013). Concepciones y creencias sobre ciencia, aprendizaje y enseñanza de profesores universitarios de ciencias. Revista Electrónica de Investigación en Educación en Ciencias, 8(1), 2441.

Cárcamo, R. y Castro, P. (2015). Concepciones sobre el aprendizaje de estudiantes de pedagogía de la universidad de Magallanes y docentes en ejercicio en la educación básica de la ciudad de Punta Arenas, Chile. Formación Universitaria, 8(5), 13-24.

Catalán, J. (2011). Psicología educacional: proponiendo rumbos, problemáticas y aportaciones. Chile: Universidad de La Serena.

Cornejo, J., Sanhueza, S. y Rioseco, M. (2012). Orientaciones para la elaboración de tesis, seminarios y papers académicos. Chile: Universidad Católica del Maule.

Díaz, V. (2006). Formación docente, práctica pedagógica y saber pedagógico. Laurus, 12(Ext.), 88-103.

Ernest, P. (1988). The impacts of beliefs on the teaching of mathematics. En C. Keitel, P. Damerow, A. Bishop y P. Gerders (Eds.), Mathematics, education and society (pp. 99-101). París: United Nations Educational Scientific.

Friz, M., Sanhueza, S., Sánchez, A., Sámuel, M. y Carrera, C. (2009). Concepciones en la enseñanza de la Matemática en educación infantil. Perfiles Educativos, 21(125), 62-73.

Friz, M., Sanhueza, S. y Figueroa, E. (2011). Concepciones de los estudiantes para profesor de Matemáticas sobre las competencias profesionales implicadas en la enseñanza de la Estadística. Revista Electrónica de Investigación Educativa, 13(2), 113-131. Recuperado de

https://redie.uabc.mx/redie/article/view/287

Gay, L. y Airasian, P. (2000). Educational research: competencies for analysis and application. Upper Saddle River, NY: Prentice-Hall.

Gamboa, R. (2014). Relación entre la dimensión afectiva y el aprendizaje de las matemáticas. Revista Electrónica Educare, 18(2), 117-139.

Gascón, J. (2001). Incidencias del modelo epistemológico de las matemáticas sobre el modelo docente. Revista Latinoamericana de Investigación en Matemática Educativa, 4(2), 129-159.

García, L., Azcárate, C. y Moreno, M. (2006). Creencias, concepciones y conocimiento profesional de profesores que enseñan cálculo diferencial a estudiantes de ciencias económicas. Revista Latinoamericana de Investigación Educativa, 9(1), 85-116. 
Gil, N., Blanco, L. y Guerrero, E. (2005). El dominio afectivo en el aprendizaje de las matemáticas. Una revisión de sus descriptores básicos. Unión, Revista Iberoamericana de Educación Matemática, 2, 15-32.

Gómez-Chacón, I. (2010). Tendencias actuales en investigación en matemáticas y afecto. En M. M. Moreno, A. Estrada, J. Carrillo y T. A. Sierra (Eds.), Investigación en educación matemática XIV (pp. 121-140). Madrid: Sociedad Española de Investigación en Educación Matemática.

Gómez-Chacón, I. (2000). Matemática emocional. Los efectos en el aprendizaje matemático. Madrid: Narcea.

Horn, I. (2008). Turnaround students in high school mathematics: constructing identities of competence through mathematical worlds. Mathematical Thinking and Learning, 10(3), 201-239.

Makuc, M. y Larrañaga, E. (2015). Teorías implícitas acerca de la comprensión de textos: Estudio exploratorio en estudiantes universitarios de primer año. Revista Signos, 48(87), 29-53.

Mato, M., Espiñeira, E. y Chao, R. (2014). Dimensión afectiva hacia la matemática: resultados de un análisis en educación primaria. Revista de Investigación Educativa, 32(1), 57-72.

Mora, F. y Barrantes, H. (2008). ¿Qué es matemática? Creencias y concepciones en la enseñanza media costarricense. Cuadernos de Investigación y Formación en Educación Matemática, 3(4), 71-81.

Ministerio de Educación. (2009). Objetivos Fundamentales y contenidos mínimos obligatorios de la educación básica y media. Santiago: Autor.

Panes, R. y Parra, V. (2013). Sistemas de creencias en las prácticas pedagógicas de profesores de matemática. Actas del VII Congreso Iberoamericano de Educación Matemática (pp. 5974-5979). Montevideo. Recuperado de http://www.cibem7.semur.edu.uy/7/actas/pdfs/687.pdf

Pehkonen, E. (2006). What do we know about teacher change in mathematics? En L. Häggblom, L. Burman y A-S. Röj-Lindberg (Eds.), Kunskapens och lärandets villkor. Festskrift tillägnad professor Ole Björkqvist [Conocimiento y condiciones de aprendizaje. Documento dedicado al profesor Ole Björkqvist] (pp. 77-87). Vasa: Abo Akademi.

Prieto, M. y Contreras, G. (2008). Las concepciones que orientan las prácticas evaluativas de los profesores: un problema a develar. Estudios Pedagógicos, 34(2), 245-262.

Sanhueza, S., Friz, M. y Quintriqueo, S. (2014). Estudio exploratorio sobre las actitudes y comportamiento del profesorado de Chile en contextos de escolarización de alumnado inmigrante. Revista Electrónica de Investigación Educativa, 16(3), 148-162. Recuperado de https://redie.uabc.mx/redie/article/view/524

Sánchez, A., Díaz, C., Sanhueza, S. y Friz, M. (2008). Percepciones y actitudes de los estudiantes de pedagogía hacia la inclusión educativa. Estudios Pedagógicos, 34(2), 169-178.

Sánchez, V., García, M., Escudero, I., Gavilán, J. M., Trigueros, R. y Sánchez-Matamoros, G. (2006). Un estudio sobre el aprendizaje de contenidos matemáticos en el Bachillerato dentro de una comunidad de indagación. En M. C. Penalva, I. Escudero y D. Barba (Eds.), Conocimiento, entornos de aprendizaje y tutorización para la formación del profesorado de Matemáticas. Granada, España: Proyecto Sur.

Sanhueza, S., Penalva, M. C. y Friz, M. (2013). Identidades y competencias profesionales de estudiantes para maestro de educación infantil relativas a la enseñanza de la geometría. Revista Latinoamericana de Investigación en Matemática Educativa, 16(1), 99-125.

Thompson, A. (1992). Teachers' beliefs and conceptions: a synthesis of the research. En G. Douglas (Ed.) Handbook of research in mathematics teaching and learning. Nueva York: Macmillan. 